\title{
THE VSOP MISSION FOR EXTRAGALACTIC
}

\section{RADIO SOURCES}

\author{
HISASHI HIRABAYASHI \\ The Institute of Space and Astronautical Science \\ 3-1-1 Yoshinodai, Sagamihara, Kanagawa 229 Japan
}

\section{VSOP System Description}

The VSOP (VLBI Space Observatory Programme) mission is being developed by the Institute of Space and Astronautical Science (ISAS), in close collaboration with the National Astronomical Observatory (NAO) of Japan. NASA and NRAO of the USA are key collaborating institutions, and most radio-telescopes world-wide will participate in some VSOP observations.

Funding for development of the VSOP satellite, MUSES-B, was first received in 1989. The satellite is scheduled for launch in September 1996. This will be just the tenth anniversary of the first successful space VLBI demonstration, the TDRSS-OVLBI experiment (Tracking and Data Relay Satellite System-Orbiting VLBI Experiment, Levy et al. 1986) at S-band. MUSES-B will place an 8 meter diameter Cassegrain radio-telescope in an elliptical Earth orbit $(22,000 \mathrm{~km}$ apogee height and $1,000 \mathrm{~km}$ perigee height, resulting in an 6.6 hour orbital period) to make the first dedicated spaceVLBI observations on baselines up to $30,000 \mathrm{~km}$. Observing frequencies are $1.6,5$ and $22 \mathrm{GHz}$, with data down-loaded in real-time on a $128 \mathrm{Mbps}$ link supported by a dedicated five station telemetry network (Hirabayashi et al. 1992; Hirosawa and Hirabayashi 1995). The ten-station VSOP correlator will be ready before the spacecraft launch.

\section{Observations of Extragalactic Radio Sources with VSOP}

On baselines to the same ground antenna, VSOP will have, at 1.6 and $5 \mathrm{GHz}$, sensitivity a factor of about 6 better than that of TDRSS-OVLBI at $2.3 \mathrm{GHz}$ (including the improvement from the larger VSOP bandwidth). 
The sensitivity of a baseline between VSOP and a VLBA antenna will be a factor of $\sim 6$ worse than between two VLBA antennas.

Because of the limited sensitivity, like the present-day VLBI, most of the observations will be limited to high brightness sources. Nearly all continuum observations will be of active galactic nuclei (AGNs) with some exceptions for possible flare stars, galactic jet sources, or supernovae events. For baselines between VSOP and VLBA antenna, the estimated number of detectable sources will be $1000 \sim 2000$ at $5 \mathrm{GHz}$ and $50 \sim 100$ at $22 \mathrm{GHz}$.

Mapping periods of 3-4 orbits will be typical for VSOP imaging observations, to build up the UV-coverage for good imaging capability. The high resolution and high dynamic range imaging capability are the most important features of the VSOP mission. Observations with one or two orbits will be used for survey observations or model-fitting type observations. Observations are classified into two categories; peer reviewed Open Program and mission-led Survey Program. The Survey Program will deal with large number of statistical samples, to be observed with a smaller number of ground telescopes.

Monitoring of structure changes will be to some degree limited by the changing UV-coverage. Both the dynamic range and the synthesized beam shape will vary with observing epoch due to the orbital precession. The constraint that sources within $70^{\circ}$ of the Sun cannot be observed results in periods when low declination sources are not observable.

\section{Science Operation of VSOP}

The Open Program is open to proposals from astronomers worldwide, and the proposals will be reviewed based on their scientific merits by an international Science Review Committee, and scheduled taking into account the resource availability. The first Announcement of Opportunity was issued in June 1995 for the first 17 months from the beginning of 1997. For details of VSOP capabilities, readers are advised to consult the VSOP Proposer's Guide, available by anonymous ftp from 133.74.2.131.

The mission's science operations will be led by the VSOP Science Operations Group (VSOG), centered at ISAS with its core members from ISAS and NAO. The scientific policy is determined by VSOP International Science Council (VISC), members of which were selected from world-wide radio-astronomical community.

\section{References}

Levy, G.S. et al. (1986) Science, 234, 187

Hirabayashi, H. et al. (1992) Proc. 18th ISTS (Kagoshima), 1813-1820

Hirosawa, H. and Hirabayashi, H. (1995) IEEE AES Systems Magazine, June, 17-23 